\title{
NF1 deficiency causes Bcl-xL upregulation in Schwann cells derived from neurofibromatosis type 1-associated malignant peripheral nerve sheath tumors
}

\author{
HO-JIN PARK ${ }^{1}$, SU-JIN LEE ${ }^{1}$, YOUNG BAE SOHN ${ }^{1}$, HYUN-SEOK JIN ${ }^{1}$, JAE-HO HAN ${ }^{2}$, \\ YOUNG-BAE KIM ${ }^{2}$, HYUNEE YIM $^{2}$ and SEON-YONG JEONG ${ }^{1}$ \\ Departments of ${ }^{1}$ Medical Genetics and ${ }^{2}$ Pathology, Ajou University \\ School of Medicine, Suwon 443-721, Republic of Korea
}

Received November 2, 2012; Accepted December 14, 2012

DOI: 10.3892/ijo.2012.1751

\begin{abstract}
Since the bi-allelic inactivation of both neurofibromin $1(\mathrm{NFl})$ gene alleles $\left(\mathrm{NFl}^{-/}\right)$in Schwann cells (SCs) is common in both benign plexiform neurofibromas (PNs) and malignant peripheral nerve sheath tumors (MPNSTs) in patients with neurofibromatosis type 1 (NF1), other genetic alterations in $\mathrm{SCs}$ may be required for tumor progression of PNs to MPNSTs. We found that the anti-apoptotic Bcl-xL protein is upregulated in MPNST tissues compared to PN tissues from patients with NF1 by immunohistological staining. In addition, we investigated whether $\mathrm{Bcl}-\mathrm{xL}$ is upregulated in SCs derived from MPNSTs and found a significantly higher Bcl-xL expression level in sNF96.2 MPNST-derived SCs compared to normal human SCs (HSCs). We also discovered that the increased $\mathrm{Bcl}-\mathrm{xL}$ expression caused an increase in drug resistance to doxorubicin in MPNST-derived SCs. Manipulation of NF1 gene expression levels by treatment with small interfering RNA (siRNA) and overexpression of the neurofibromin GAP-related domain (NF1-GRD) demonstrated that upregulated Bcl-xL expression in MPNST-derived SCs was caused by NF1 deficiency. Treatment with the Erk1/2 inhibitor, PD98059, resulted in a slight increase in Bcl-xL levels in neurofibromindepleted normal HSCs, indicating that Bcl-xL upregulation in MPNST-derived SCs is mediated by activated Erk1/2, which is a Ras downstream protein regulated by neurofibromin. As the reduction of Bcl-xL expression restored sensitivity to
\end{abstract}

Correspondence to: Dr Seon-Yong Jeong, Department of Medical Genetics, School of Medicine, Ajou University, San 5, Wonchon dong, Yeongtong-gu, Suwon 443-721, Republic of Korea

E-mail: jeongsy@ajou.ac.kr

Dr Hyunee Yim, Department of Pathology, School of Medicine, Ajou University, San 5, Wonchon dong, Yeongtong-gu, Suwon 443-721, Republic of Korea

E-mail: snoopy@ajou.ac.kr

Key words: neurofibromatosis type 1, malignant peripheral nerve sheath tumor, Schwann cells, apoptosis, doxorubicin, ABT-737 doxorubicin-induced apoptosis in sNF96.2 cells, we examined the effect of the small molecule Bcl-xL inhibitor ABT-737 on sNF96. 2 cells. A very low dose of ABT-737 combined with doxorubicin synergistically enhanced sensitivity to doxorubicin-induced apoptosis in sNF96.2 cells, suggesting that ABT-737 and doxorubicin may be a good combination to effectively treat NF1-associated MPNSTs with minimal side-effects. Collectively, our results suggest that upregulation of Bcl-xL in MPNST-derived SCs may be caused by the NF1 deficiency-mediated elevation in Ras/MAPK signaling and may provide a new potential chemotherapeutic target in patients with NF1 and MPNSTs.

\section{Introduction}

Neurofibromatosis type 1 (NF1; MIM \#162200) is a commonly inherited autosomal dominant disorder characterized by variable phenotypic features, including cutaneous manifestations, such as café au lait spots, neurofibromas and freckling of the axillary or inguinal regions, as well as extracutaneous manifestations such as Lisch nodules, optic nerve gliomas, scoliosis, bone dysplasia, malignant tumors, and cognitive impairment $(1,2)$. NF1 is caused by neurofibromin 1 (NF1) gene mutations, which encode neurofibromin, a GTPase-activating protein (GAP) (3). The majority of patients with NF1 develop benign dermal neurofibromas (DNs) and/or plexiform neurofibromas (PNs) $(4,5)$.

Neurofibromas are composed of a mixture of cell types including Schwann cells (SCs), fibroblast cells, mast cells, and perineural cells (6). SCs are believed to be the primary pathogenic cell source in neurofibromas (7). As the complete loss of the NFI gene has been identified exclusively in neurofibroma SCs (8-10) and the loss of NF1 in the SC lineage is sufficient to generate tumors in mice (11), the bi-allelic inactivation of both $\mathrm{NF} 1$ alleles $\left(\mathrm{NF}^{-/}\right)$in SCs by germline $\mathrm{NF1}$ mutation at one allele and the additional somatic loss of heterozygosity $(\mathrm{LOH})$ at the remaining functional NF1 locus, has been suggested to be a major cause of NF1 tumorigenesis. In addition, haploinsuffiency in other types of cells $\left(\mathrm{NFI}^{+/}\right)$in neural crest-derived tissues, including fibroblast cells, mast cells and perineurial cells also plays an important role in the pathoetiology of NF1 $(6,12,13)$. 
Malignant peripheral nerve sheath tumors (MPNSTs) are a type of aggressive sarcoma and are a major cause of mortality in patients with NF1 (5,14-16). The lifetime risk of developing MPNSTs in patients with NF1 is $8-13 \%$ (17) or $5.9-10.3 \%$ (18). The majority of NF1-associated MPNSTs (approximately $85 \%$ of cases) are high-grade malignant tumors. The malignant transformation of benign PNs to MPNSTs in patients with NF1 is notable (19) and is of far greater concern to patients with NF1 (20); however, the pathogenesis is poorly understood. The bi-allelic inactivation of the NFl gene caused by a germline first-hit mutation and a somatic second-hit LOH in SCs has been identified in DNs $(21,22)$, PNs $(23,24)$, and MPNSTs $(24,25)$ in patients with NF1, indicating that the complete loss of the $N F 1$ gene $\left(N F 1^{-/-}\right)$in SCs contributes to benign neurofibroma formation and progression to MPNSTs. Since bi-allelic inactivation of the NFl gene is insufficient to explain the pathogenesis of tumor progression in NF1, cooperating genetic or epigenetic changes have been suggested to be involved in MPNST pathogenesis. Hence, robust histological and molecular analyses have been conducted to compare neurofibromas and MPNSTs $(15,26,27)$, and recently developed genome-wide DNA copy number change profiling using array comparative genomic hybridization has identified causal genes in MPNST development $(28,29)$. To date, genes involved in regulating the cell cycle and growth signal transduction have been reported mainly to be dysregulated in MPNSTs $(6,30,31)$. A number of studies have focused on genetic alterations in SCs, as most MPNSTs are thought to arise from SCs $(7,32)$.

We unexpectedly found that the anti-apoptotic protein, $\mathrm{Bcl}-\mathrm{xL}$, is upregulated in primary-cultures and established NF1-associated MPNST cells. Bcl-xL is responsible for the acquired anticancer drug resistance of MPNST cells (33). In this study, we compared Bcl-xL expression levels between normal and MPNST-derived SCs, as well as between PNs and MPNST tissues from patients with NF1 to determine whether Bcl-xL upregulation in SCs is involved in MPNST pathogenesis. Furthermore, we also examined changes in Bcl-xL expression levels and sensitivity to apoptosis induced by anticancer drugs in $\mathrm{NFI}^{+/+}$and $\mathrm{NF}^{-/-}$SCs when $\mathrm{NFl}$ expression was manipulated to determine whether $\mathrm{Bcl}-\mathrm{xL}$ upregulation is associated with NF1 deficiency in SCs.

\section{Materials and methods}

Antibodies and reagents. Anti-Bcl-xL, anti-Bcl2, anti-Bax, anti-caspase 3, anti-extracellular signal-regulated kinase (Erk)1/2 and anti-phosphorylated Erk1/2 antibodies were purchased from Cell Signaling Technology (Danvers, MA, USA). Anti-neurofibromin, anti- $\alpha$-tubulin, anti-p53, antiMcl-1, HRP-conjugated goat anti-rabbit IgG and HRPconjugated goat anti-mouse $\mathrm{IgG}$ antibodies were purchased from Santa Cruz Biotechnology (Santa Cruz, CA, USA), and anti-S100 and anti-GFP antibodies were purchased from Thermo Scientific Pierce (Rockford, IL, USA) and Invitrogen (Carlsbad, CA, USA), respectively. Doxorubicin and PD98059 were obtained from Sigma-Aldrich (St. Louis, MO, USA). ABT-737 was purchased from Santa Cruz Biotechnology.

Cell culture. Human normal Schwann cells (HSCs) and Schwann cell-like MPNST cells (sNF96.2) were purchased from ScienCell Research Laboratories (Carlsbad, CA, USA) and the American Type Culture Collection (Manassas, VA, USA), respectively. Cells were cultured in Dulbecco's modified Eagle medium (HyClone Laboratories, Logan, UT, USA) containing $10 \%$ fetal bovine serum supplemented with penicillin $(100 \mathrm{U} / \mathrm{ml})$ and streptomycin $(100 \mu \mathrm{g} / \mathrm{ml})$ at $37^{\circ} \mathrm{C}$ under a humidified atmosphere containing $5 \% \mathrm{CO}_{2}$.

Hematoxylin and eosin $(H \& E)$ staining. Tumor tissues were obtained from patients with NF1 by surgical resection. The specimens were formalin-fixed and embedded in paraffin wax for pathological evaluation by routine light microscopy. Serial $3-\mu \mathrm{m}$ sections were prepared on glass using a cryostat, and the slides were stained with H\&E.

Immunohistochemistry (IHC). Formalin-fixed paraffinembedded (FFPE) blocks from six patients with NF1 were cut at $10 \mu \mathrm{m}$, and the sections were dewaxed, rehydrated, followed by antigen retrieval in boiling citrate buffer. Immunostaining was carried out using an Ultravision LP-HRP Polymer DAB kit (Thermo Fisher Scientific, Kalamazoo, MI, USA), according to the manufacturer's instructions. Briefly, the sections were incubated with Ultra V Block (Lab Vision, Kalamazoo, MI, USA) for $5 \mathrm{~min}$ at room temperature to reduce the non-specific background, and were then treated with hydrogen peroxide to block endogenous peroxidase activity. The sections were incubated with primary antibody for $1 \mathrm{~h}$ and then incubated with HRP polymer for $20 \mathrm{~min}$. The reaction product was visualized with DAB chromogen. Pathological evaluation was performed under light microscopy. The present study using human FFPE samples was approved by the Institutional Review Board of the Ajou University School of Medicine, Suwon, Korea.

Plasmid constructs and small interfering RNAs (siRNAs). Plasmid constructs encoding wild-type Bcl-xL were generated as described previously (34). The cDNA of the GAP-related domain (GRD) region (1,181 bp) was amplified by reverse transcription-polymerase chain reaction (RT-PCR) using the primers to generate the plasmid construct expressing the human NFl-GRD: 5'-ATAGATCTACCATGGATCTCCAGA CAAGAGCTACATTTATG-3' and 5'-GTAAGCTTAACCAG TGTGTATCTGCCACAGGT-3', from total RNAs of human skin tissue cultured fibroblast cells. The cDNAs were subcloned into the pEYFP-C1 vector (Clontech, Palo Alto, CA, USA) using the BglII and HindIII restriction enzyme sites. The siRNAs were synthesized by Genolution Pharmaceuticals, Inc. (Seoul, South Korea). The target sequences for the siRNAs were as follows: 5'-CAGTGAACGTAAGGGTTCT-3' for the NF1 gene, 5'-CAGGGACAGCATATCAGAG-3' for the BCL2L1 (Bcl-xL) gene and 5'-CCTACGCCACCAATTTCGT-3' for the non-specific negative control. Cell transfection of the siRNAs and plasmid constructs was conducted using Lipofectamine RNAiMAX (Invitrogen) and Lipofectamine 2000 (Invitrogen), respectively, according to the manufacturer's instructions.

Cell viability assay. Cell viability was assessed using the EZ-Cytox Cell Viability Assay kit (Daeil Labservice, Seoul, Korea). Cells were seeded in a 96 -well tissue-culture plate $\left(7 \times 10^{3}\right.$ cells/well), cultured overnight, and then treated with various concentrations of doxorubicin and/or ABT-737. After $24 \mathrm{~h}$ of 
incubation, $10 \mu \mathrm{l}$ of Ez-Cytox reagent was added to each well, and the cells were incubated for a further $2 \mathrm{~h}$. The plate was read with an enzyme-linked immunosorbent assay microplate reader (Bio-Rad Model 680; Hercules, CA, USA) at $450 \mathrm{~nm}$.

Real-time RT-PCR. Total RNAs were isolated from the cultured cells using TRIzol reagent (Invitrogen), treated with RNase-free DNase I (Invitrogen) to avoid amplification of genomic DNA, and were subsequently reverse-transcribed using the RevertAid ${ }^{\mathrm{TM}} \mathrm{H}$ Minus First-Strand cDNA Synthesis kit (Fermentas, Burlington, ON, Canada) with the oligo(dT) ${ }_{15-18}$ primer. Real-time RT-PCR was performed using the SYBRGreen I qPCR kit (Takara, Shiga, Japan). The specific primers used were as follows: 5'-GTCGGATCGCAGCTTGGATGG CCAC-3' and 5'-CGTCAGGAACCAGCGGTTGAAGCGT-3' for $B C L 2 L 1$. The P238284 primer set (Bioneer, Seoul, Korea) was used for NF1 and 5'-TGTTGCCATCAATGACCCCTT-3' and 5'-CTCCACGACGTACTCAGCG-3' for the GAPDH gene (a relative quantification standard). All real-time RT-PCR measurements were performed using the ABI Prism 7500 Fast Real-time PCR System (Applied Biosystems, Foster City, CA, USA).

Western blot analysis. Cultured cells were lysed in RIPA buffer [150 mM NaCl, $1 \%$ Nonidet P-40, $0.5 \%$ sodium deoxycholate, $0.1 \%$ sodium dodecyl sulfate (SDS), and $50 \mathrm{mM}$ Tris buffer, $\mathrm{pH}$ 8.0]. Proteins were heated at $100^{\circ} \mathrm{C}$ for $10 \mathrm{~min}$ and analyzed by SDS-polyacrylamide gel electrophoresis on 8-12\% polyacrylamide gels. The proteins were electroblotted onto PVDF membranes (Millipore, Milford, MA, USA). The membrane blots were blocked with $5 \%(\mathrm{w} / \mathrm{v})$ non-fat dried milk, incubated with primary and secondary antibodies, and then visualized with the Enhanced Chemiluminescence Western Blotting Detection System (WEST-ZOL plus; iNtRON Biotechnology, Daejeon, Korea).

Statistical analysis. In this study, the results are expressed as the means \pm standard deviation. All experiments were repeated at least three times. Statistical significance was determined by the two-tailed Student's t-test, and P-values $<0.05$ were considered to indicate statistically significant differences.

\section{Results}

Higher Bcl-xL expression observed in MPNSTs compared to PNs in patients with NF1. Since Bcl-xL hyperexpression has been observed in NF1-associated MPNST cells (33), we confirmed this finding in the tumor tissues of patients with NF1. Tumor specimens were obtained by surgical resection from six patients with NF1. The patients were diagnosed with NF1 at the Ajou University Hospital according to NF1 diagnostic criteria (35). The clinical features of the patients are summarized in Table I. Histopathological analysis of the tumor specimens by H\&E staining revealed that three patients [patient (P)1-P3] had benign PNs and three patients (P4-P6) had MPNSTs (Table I). The H\&E results of P1 and P4 are shown in Fig. 1. The PNs were composed of loosely spaced tumor cells in a myxoid matrix or collagenous strands, whereas MPNSTs showed densely cellular atypical spindle cells forming intersecting fascicles. We then compared the Bcl-xL

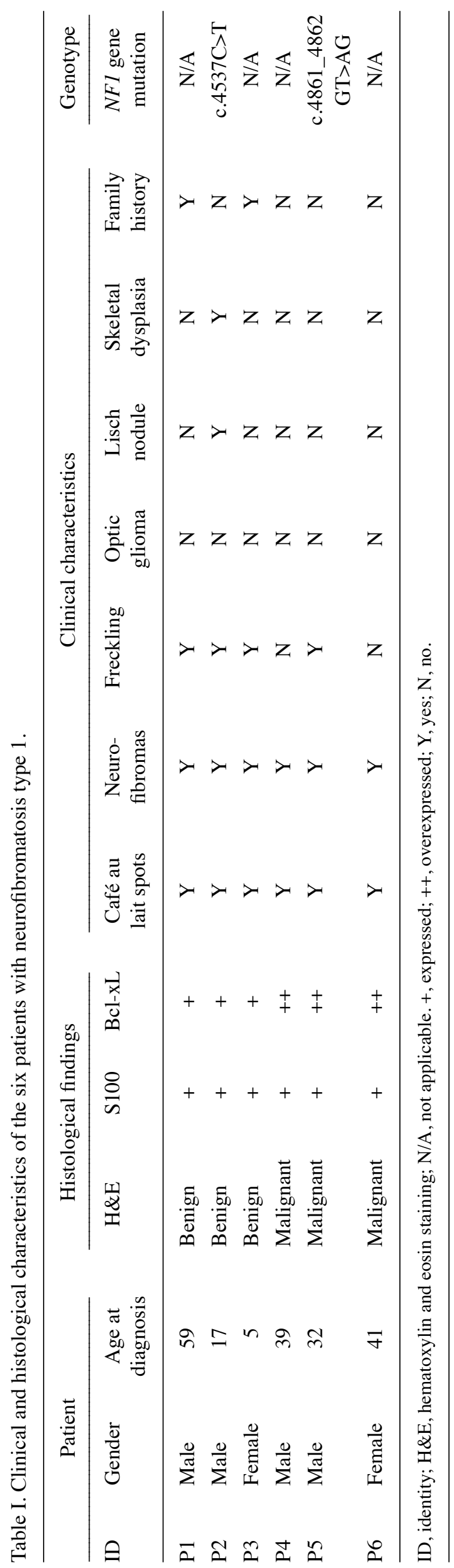



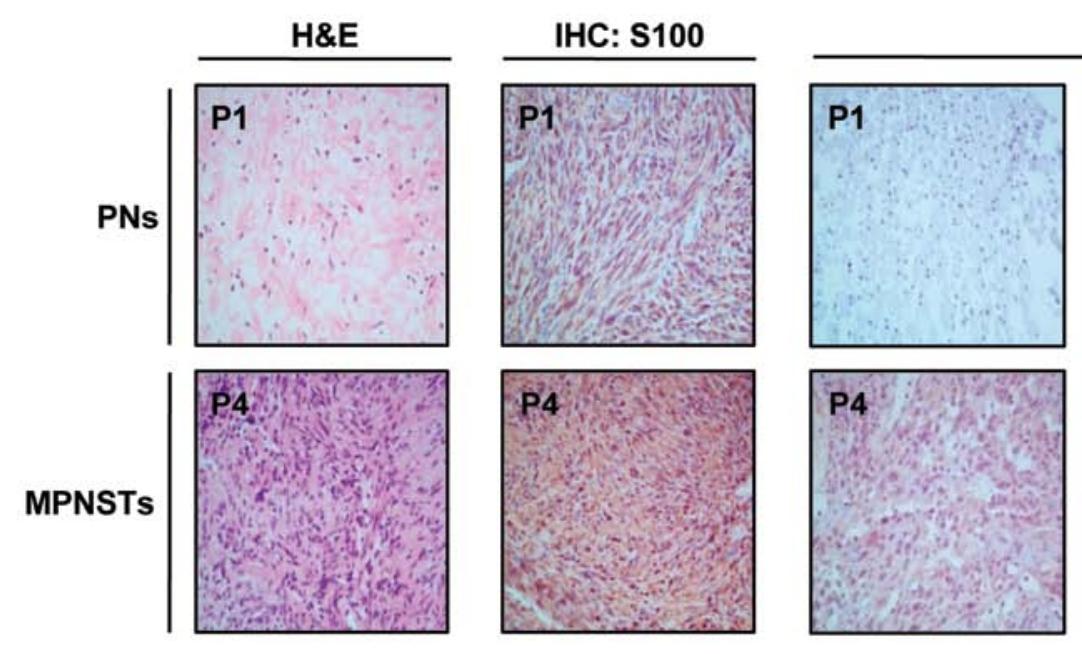

IHC: Bcl-xL
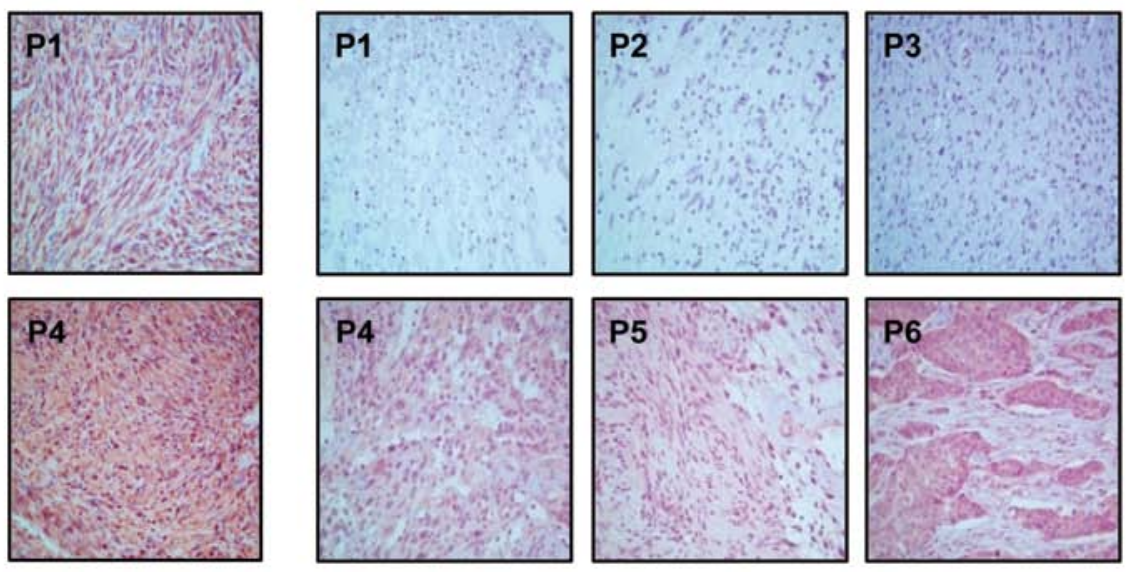

Figure 1. Immunohistochemical staining (IHC) of Bcl-xL in the plexiform neurofibroma (PN) and malignant peripheral nerve sheet tumor (MPNST) tissues from patients with neurofibromatosis type 1 (NF1). Histological analysis by standard hematoxylin and eosin (H\&E) staining and IHC using antibodies against S100 and Bcl-xL were carried out on PN and MPNST tissue sections.

expression levels between the PN and MPNST tumor tissues by IHC analysis. The IHC evaluation revealed a higher Bcl-xL expression in MPNSTs (P4-P6) compared to PNs (P1-P3) in all patients with NF1 tested.

SCs are generally thought to be the major progenitors of neurofibromas and MPNSTs and characteristically express the S100 protein (36). IHC staining using an antibody against the SC lineage marker, S100, showed that all tumors contained S100-positive cells (Table I) and more S100-positive cells were present in MPNSTs than in PNs, as shown in P1 and P4 (Fig. 1). These results suggest that tumors from patients with NF1 mainly originate from SCs and that most Bcl-xLoverexpressing cells are SC lineage cells.

Upregulation of $B c l-x L$ causes an increase in resistance to doxorubicin in NF1-deficient MPNST SCs. We examined whether the basal Bcl-xL expression levels were different between SCs derived from normal tissues and those derived from MPNSTs. We compared the normal human SC line (HSC) and the sNF96.2 SC line, which was derived from a MPNST in a patient with NF1. HSCs have both normal NF1 alleles $\left(\mathrm{NFI}^{+/+}\right)$, whereas the sNF96.2 cells have a complete $\mathrm{LOH}$ and no remaining $N F 1$ allele $\left(N F 1^{-/}\right)$(37). Western blot analysis for neurofibromin confirmed normal neurofibromin expression in the HSCs and null neurofibromin expression in the sNF96.2 cells (Fig. 2A). The increased pErk1/2 protein level involved in the Ras/Raf/Mek/Erk signaling pathway (38), demonstrated that the sNF96.2 cells were MPNST-derived SCs (Fig. 2A). Basal Bcl-xL expression was significantly upregulated in the sNF96.2 cells compared to the HSCs, whereas the lower expression of $\mathrm{Bcl}-2$, another anti-apoptotic protein, was observed in the sNF96.2 cells (Fig. 2A). No changes in Mcl-1 and p53 expression levels were observed between the two cell lines.

Chemoresistance in NF1-associated MPNST cells has been recently reported (33). We examined sensitivity to apoptosis induced by anticancer drugs in HSCs and sNF96.2 cells to determine whether SCs are responsible for chemoresistance in MPNSTs. We used doxorubicin as doxorubicin has been suggested to be a good candidate for MPNST chemotherapy $(33,39)$. The cell viability assay results demonstrated that the sNF96.2 cells were more resistant to doxorubicin than the HSCs (Fig. 2B), suggesting that the upregulation of Bcl-xL may decrease apoptosis sensitivity in SNF96.2 cells.

Bcl-xL expression level is closely related to sensitivity to doxorubicin-induced apoptosis in normal SCs and NF1-deficient MPNST-derived SCs. We manipulated Bcl-xL expression levels in HSCs and sNF96.2 cells to determine whether the sensitivity to doxorubicin-induced apoptosis in SCs was dependent on the $\mathrm{Bcl}-\mathrm{xL}$ expression level. The overexpression of $\mathrm{Bcl}-\mathrm{xL}$ in HSCs decreased caspase 3 cleavage activity significantly and increased cell viability following doxorubicin treatment (Fig. 3A and B). By contrast, the downregulation of Bcl-xL in the sNF96.2 cells following treatment with siRNAs targeting the BCL2L1 gene significantly increased caspase 3 cleavage activity and reduced cell viability following doxorubicin treatment (Fig. 3C and D). These results indicate that the $\mathrm{Bcl}-\mathrm{xL}$ level is closely related to sensitivity to doxorubicin-induced apoptosis in both types of SC (HSCs and MPNST-derived SCs).

Bcl-xL expression level is mediated by NF1 gene level in normal SCs and NF1-deficient MPNST-derived SCs. The genetically noticeable difference between HSCs and sNF96.2 cells is determined by whether the $N F 1$ gene is intact $\left(\mathrm{NFI}^{+/+}\right)$ or inactivated $\left(\mathrm{NFI}^{-/}\right)$, suggesting that the hyperexpression of Bcl-xL in sNF96.2 cells may be mediated by decreased NF1 expression. We manipulated neurofibromin expression levels in HSCs and sNF96.2 cells to determine whether Bcl-xL expression is dependent on neurofibromin expression in SCs. The downregulation of neurofibromin expression by siRNA targeting the $\mathrm{NFl}$ gene in HSCs caused an increase in Bcl-xL expression and pErk1/2 protein levels, but had no effect on other apoptosis-related proteins such as Bcl-2, Mcl-1, Bax and p53 (Fig. 4A). Real-time PCR results of BCL2L1 demonstrated that the increased $\mathrm{Bcl}-\mathrm{xL}$ expression level in the neurofibromindepleted HSCs was caused by the increased BCL2L1 mRNA expression (Fig. 4B). The neurofibromin-depleted HSCs 
A

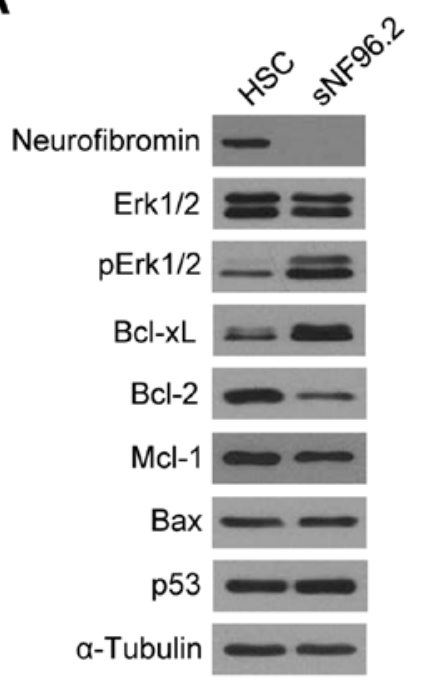

B

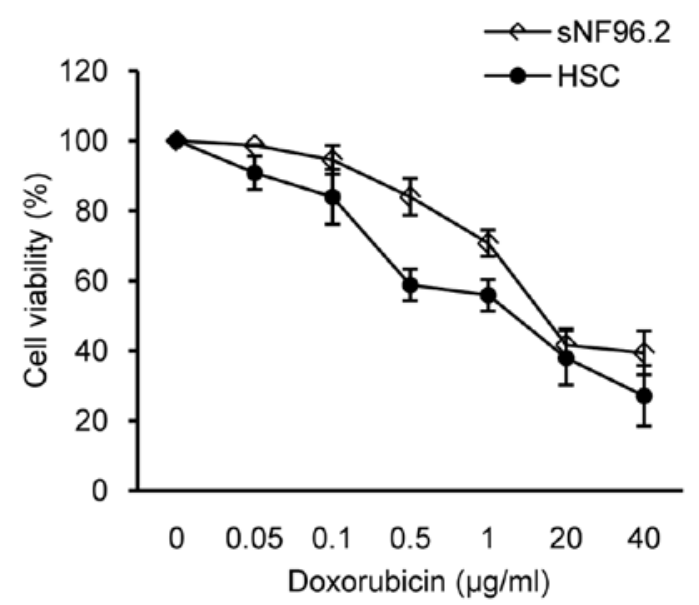

Figure 2. Comparison of a normal human Schwann cell line (HSC) and human NF1-depleted Schwann cell line characteristics (sNF96.2). (A) Endogenous protein levels of extracellular signal-regulated kinase (Erk)1/2, phosphorylated Erk1/2 (pErk1/2), Bcl-xL, Bcl-2, Mcl-1, Bax, p53 and $\alpha$-tubulin in the HSC and sNF96.2 cells were assessed by western blot analysis. The $\alpha$-tubulin protein level was used as the internal control. (B) Sensitivities to apoptosis induced by the anticancer drug, doxorubicin, in HSCs and sNF96.2 cells were measured by cell viability assay. The HSCs and sNF96.2 cells were treated with the indicated concentrations of doxorubicin, and cell viability was determined by the Ez-Cytox assay after $24 \mathrm{~h}$ of incubation.

A

$\underline{\text { HSC }}$

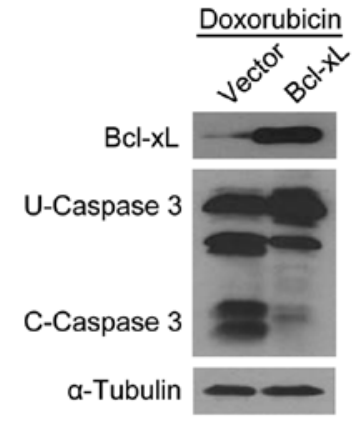

C

SNF96.2
B

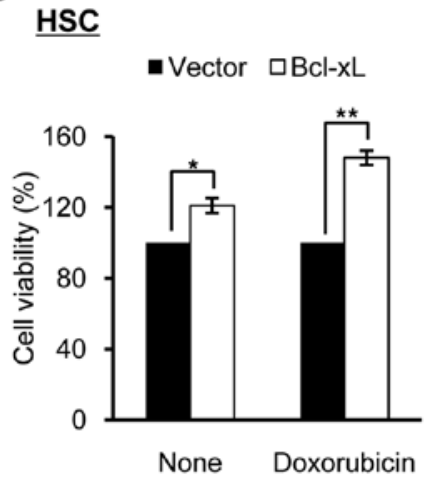

D SNF96.2

- Control siRNA $\square$ BCL2L1 siRNA

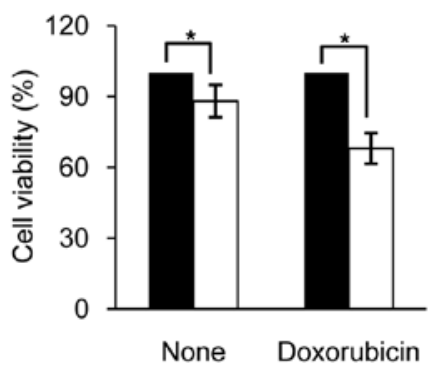

Figure 3. Bcl-xL level-dependent alteration of sensitivity to doxorubicin-induced apoptosis in the normal human Schwann cell line (HSC) and human NF1-depleted Schwann cell line (sNF96.2). (A and B) Overexpression of Bcl-xL in HSCs. The HSCs were transfected with pcDNA3.1 plasmid vector or a Bcl-xL plasmid construct and then incubated for $24 \mathrm{~h}$. The cells were further treated with $0.5 \mu \mathrm{g} / \mathrm{ml}$ doxorubicin for $24 \mathrm{~h}$. (C and D) Knockdown of Bcl-xL using small interfering RNAs (siRNAs) in sNF96.2 cells. sNF96.2 cells were treated with BCL2L1 gene siRNAs (50 nM) or the non-specific negative control $(50 \mathrm{nM}$ ) and incubated for $72 \mathrm{~h}$. The cells were further treated with $0.5 \mu \mathrm{g} / \mathrm{ml}$ doxorubicin for $24 \mathrm{~h}$. (A and C) Protein levels of Bcl-xL, uncleaved caspase 3 (U-caspase 3), cleaved caspase 3 (C-caspase 3), and $\alpha$-tubulin were determined by western blot analysis. The $\alpha$-tubulin protein level was used as the internal control. (B and D) Cell viabilities of the untreated (None) and doxorubicin treated (Doxorubicin) cells that were transfected with control or BCL2L1 siRNAs were determined by the Ez-Cytox assay. ${ }^{*} \mathrm{P}<0.05$ and $^{* *} \mathrm{P}<0.01$. 
A

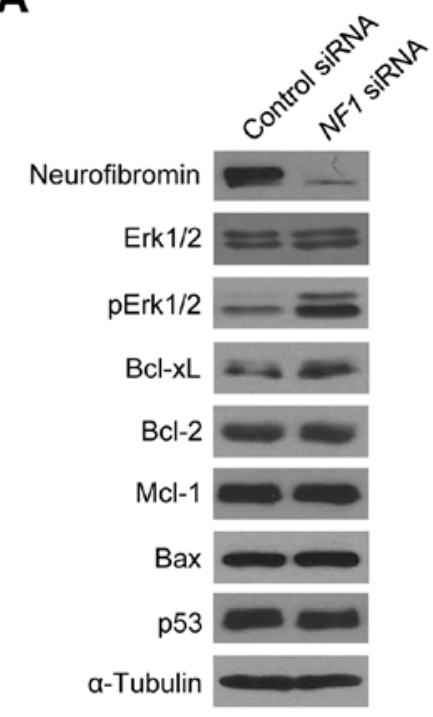

C

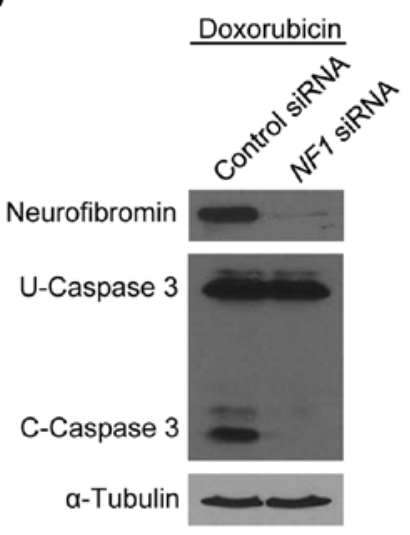

B

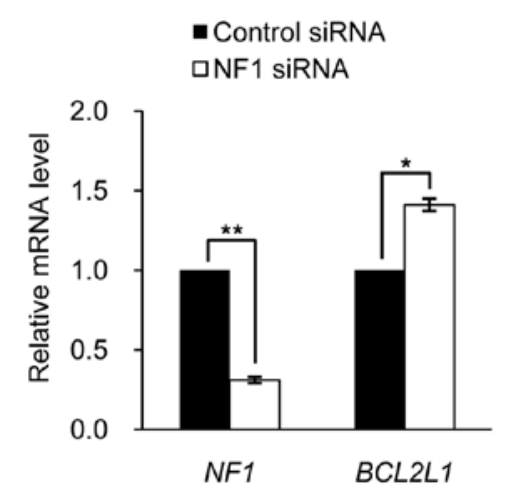

D

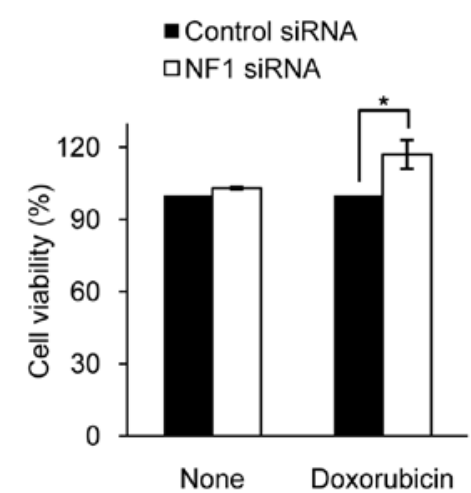

Figure 4. An increase in Bcl-xL expression and a decrease in sensitivity to doxorubicin-induced apoptosis in the normal human Schwann cell line (HSC) by NF1 knockdown. (A) The HSCs were transfected with NF1 gene siRNAs (100 nM) or the non-specific negative control (100 nM) and then incubated for $72 \mathrm{~h}$. Protein levels of extracellular signal-regulated kinase (Erk)1/2, phosphorylated Erk1/2 (pErk1/2), Bcl-xL, Bcl-2, Mcl-1, Bax, p53 and $\alpha$-tubulin in the HSCs were assessed by western blot analysis. The $\alpha$-tubulin protein level was used as the internal control. (B) Relative NF1 and BCL2L1 mRNA levels in HSCs transfected with control or NF1 siRNAs and assessed by real-time reverse transcription-polymerase chain reaction (RT-PCR). ${ }^{* *} \mathrm{P}<0.01$. (C and D) The HSCs transfected with control or NF1 siRNAs were further treated with $0.5 \mu \mathrm{g} / \mathrm{ml}$ doxorubicin for $24 \mathrm{~h}$. Protein levels of neurofibromin, uncleaved caspase 3 (U-caspase 3 ), cleaved caspase 3 (C-caspase 3), and $\alpha$-tubulin were determined by western blot analysis. The $\alpha$-tubulin protein level was used as the internal control. (D) Cell viabilities of the untreated (None) and doxorubicin treated (Doxorubicin) cells transfected with control or NF1 siRNAs were determined by the Ez-Cytox assay. ${ }^{*} \mathrm{P}<0.05$.

showed decreased caspase 3 cleavage activity and increased cell viability following doxorubicin treatment for $24 \mathrm{~h}$ after $72 \mathrm{~h}$ of NF1 siRNA treatment (Fig. 4C and D), similar to the Bcl-xL-overexpressing HSCs (Fig. 3A and B).

We then overexpressed NF1 in sNF96.2 cells. Since the $N F 1$ gene is very large, and the NF1-GRD is sufficient to restore normal growth in mouse $N F 1^{-/}$cells (40), we constructed a human NF1-GRD fused to GFP. NF1-GRD-GFP overexpression in the sNF96.2 cells significantly increased caspase 3 cleavage activity and reduced cell viability following doxorubicin treatment (Fig. 5A and B), similar to the Bcl-xL-depleted sNF96.2 cells (Fig. 3C and D). These results indicate that Bcl-xL expression level is mediated by the NF1 gene level in both types of SC (HSCs and MPNST-derived SCs).

NF1 deficiency induces Bcl-xL expression by activating Erk1/2 in the Ras/mitogen-activated protein kinase (MAPK) signaling pathway. We then wished to clarify the molecular mechanisms by which alterations in neurofibromin expression in SCs modulate the Bcl-xL expression level. As shown in Fig. 2A, Erk1/2 was highly activated in the sNF96.2 cells when Bcl-xL was highly expressed, suggesting that the activation of the Erk1/2 downstream effector in the Ras/MAPK signaling pathway may be involved in NF1 deficiency-mediated Bcl-xL upregulation in MPNST-derived SCs. We first examined whether the inhibition of Erk1/2 could influence the Bcl-xL expression level in sNF96.2 cells. When the sNF96.2 cells were treated with the Erk1/2 inhibitor, PD98059, for $24 \mathrm{~h}$, the Bcl-xL protein level decreased in a dose-dependent manner (Fig. 6A). Subsequently, the neurofibromin-depleted HSCs following transfection with NF1 siRNAs exhibited a significant increase in pErk1/2 and Bcl-xL levels in the absence of PD98059; however, the neurofibromin-depleted HSCs showed a slight increase in pErk1/2 and Bcl-xL levels in the presence 
A

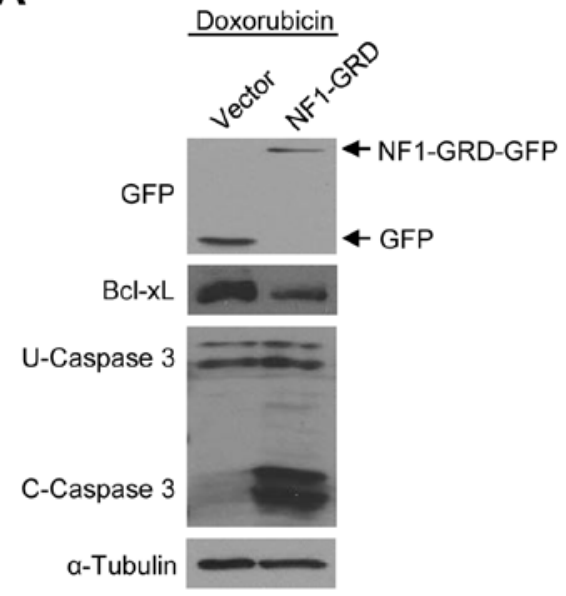

B

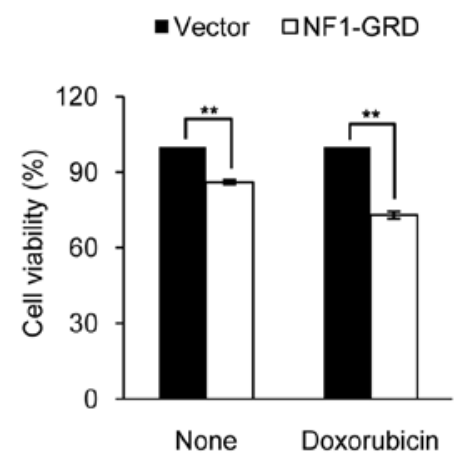

Figure 5. Enhanced sensitivity to doxorubicin-induced apoptosis in the human NF1-depleted Schwann cell line (sNF96.2) by NF1-GRD overexpression. sNF96.2 cells were transfected with the pEGFP-C1 vector or the NF1-GRD plasmid construct and then incubated for $24 \mathrm{~h}$. The normal human Schwann cells (HSCs) were further treated with $0.5 \mu \mathrm{g} / \mathrm{ml}$ doxorubicin for $24 \mathrm{~h}$. (A) Protein levels of uncleaved caspase 3 (U-caspase 3 ), cleaved caspase 3 (C-caspase 3 ) and $\alpha$-tubulin were determined by western blot analysis. The $\alpha$-tubulin protein level was used as the internal control. (B) Cell viabilities of the untreated (None) and doxorubicin treated (Doxorubicin) cells transfected with the pEGFP-C1 vector or the NF1-GRD plasmid construct were determined by the Ez-Cytox assay. ${ }^{* *}$ P 0.01 .

A

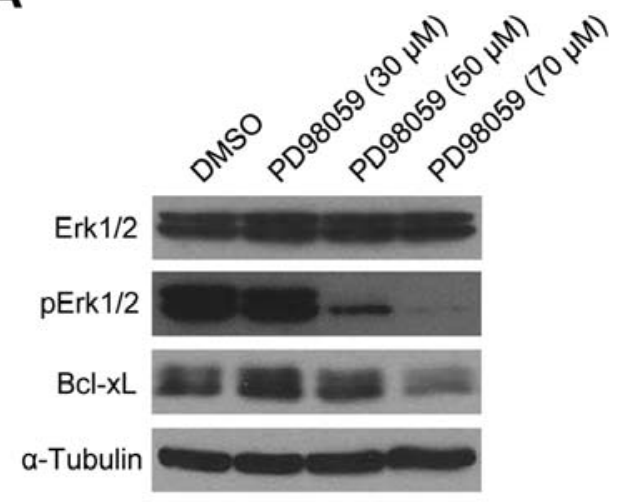

B

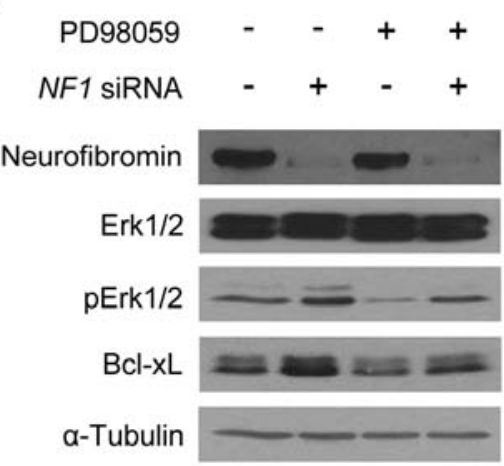

Figure 6. Extracellular signal-regulated kinase (Erk)1/2-mediated upregulation of Bcl-xL in NF1-deficient Schwann cells. (A) Human NF1-depleted Schwann cells (sNF96.2) were treated with DMSO (control) or the indicated concentrations of the Erk1/2 inhibitor, PD98059, for 24 h. Protein levels of Erk1/2, phosphorylated Erk1/2 (pErk1/2), Bcl-xL and $\alpha$-tubulin in sNF96.2 cells were assessed by western blot analysis. The $\alpha$-tubulin protein level was used as the internal control. (B) Normal human Schwann cells (HSCs) that were transfected with NF1 siRNAs (100 nM) or the non-specific negative control siRNA (100 nM) for $72 \mathrm{~h}$ were treated with DMSO or $70 \mu \mathrm{M}$ PD98059 and then incubated for $24 \mathrm{~h}$. Protein levels of Erk1/2, phosphorylated Erk1/2 (pErk1/2), Bcl-xL and $\alpha$-tubulin in the HSCs were assessed by western blot analysis. The $\alpha$-tubulin protein level was used as the internal control.

of PD98059 (Fig. 6B). These results suggest that the Erk1/2 activation level may play a crucial role in the $N F 1$ dosedependent Bcl-xL expression changes in both SCs.

The Bcl-xL inhibitor, ABT-737, synergistically enhances sensitivity to doxorubicin-induced apoptosis in NF1-deficient MPNST-derived SCs. Chemotherapy for NF1-associated MPNSTs has not been extensively investigated. ABT-737, a mimetic of the $\mathrm{BH} 3$-only protein $\mathrm{Bad}$ and which binds selectively to Bcl-2, Bcl-xL and Bcl-w (41), induces synergistic cytotoxicity in MPNST cells when combined with doxorubicin (33). We then investigated whether ABT-737 would exert a synergistic cytotoxic effect in sNF96.2 cells when used in combination with doxorubicin. As a result, ABT-737 effectively enhanced apoptotic cell death in a dose-dependent manner, compared to the cells treated with doxorubicin alone at both concentrations of doxorubicin ( 0.1 and $0.5 \mu \mathrm{g} / \mathrm{ml})$ (Fig. 7). The concentrations of ABT-737 and doxorubicin required for $50 \%$ cytotoxicity in the sNF96.2 and MPNST-derived SCs were calculated to be $0.1 \mu \mathrm{M}$ ABT-737 plus $0.5 \mu \mathrm{g} / \mathrm{ml}$ doxorubicin.

\section{Discussion}

Mouse model studies have reported that the double-hit inactivation of the $\mathrm{NFl}$ gene $\left(\mathrm{NFI}^{--}\right)$in SCs leads benign DNs and/or PNs to form tumors (42). Other genetic alterations in SCs may be required for tumors to progress from PNs to MPNSTs. As lossof-function mutations in tumor suppressor genes, such as TP53, $R B 1$ and $C D K N 2 A$ are particularly common in MPNSTs (31), the accumulation of additional loss-of-function mutations in these tumor suppressor genes in $\mathrm{NFI}^{-/-} \mathrm{SCs}$ may be required for MPNST pathogenesis. In addition, the dysregulation of many 


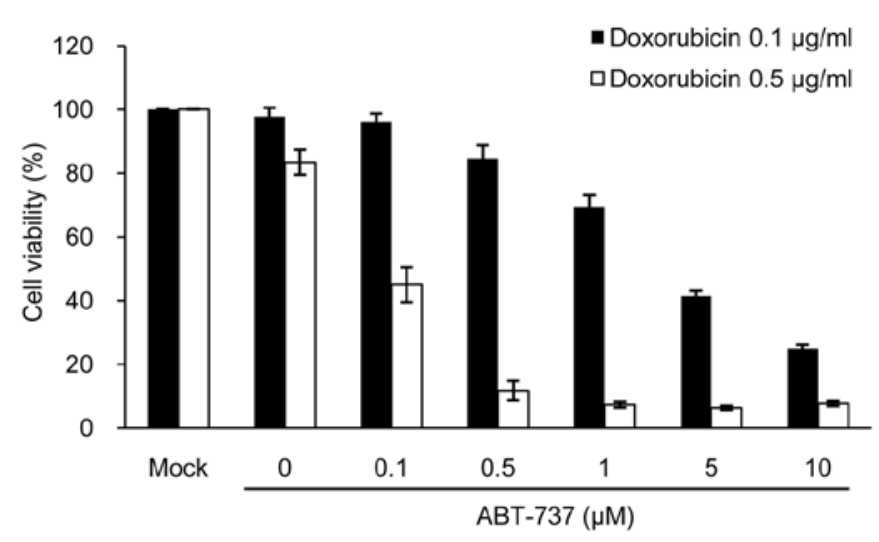

Figure 7. Synergistic effect of ABT-737 on sensitivity to doxorubicin-induced apoptosis in the human NF1-depleted Schwann cell line (sNF96.2). sNF96.2 cells were treated with 0.1 or $0.5 \mu \mathrm{g} / \mathrm{ml}$ doxorubicin alone or co-treated with the indicated concentrations of ABT-737. The doxorubicin non-treated cells (Mock) were used as the control. After $24 \mathrm{~h}$ incubation, cell viabilities were determined by the Ez-Cytox assay.

genes in MPNSTs has been reported (7,31). In particular, a large-scale comparison between human MPNST-derived SCs and normal SCs revealed a relative downregulation of the SC differentiation markers, SOX10, CNP and PMP22, and nerve growth factor receptor, as well as the relative upregulation of the neural crest stem cell markers, SOX9 and TWIST1, in MPNST-derived SCs (27). Post-transcriptional modification by microRNAs has also been studied in NF1 and the results showed the upregulation of miR-10b (43) and the downregulation of miR34a whose expression is mediated by p53 (44) in MPNST-derived SCs or tissues.

We recently reported the hyperexpression of the anti-apoptotic protein, Bcl-xL, in primary MPNST cells and a MPNST cell line (33). Hence, in this study, we aimed to confirm this result in patient samples as a first step and demonstrated the upregulation of Bcl-xL in MPNSTs from patients with NF1 by Bcl-xL immunohistological staining (Fig. 1). As most Bcl-xL expressing cells were S100-positive, we investigated Bcl-xL upregulation in the SCs. As expected, we found a higher Bcl-xL expression in the sNF96.2 MPNST-derived SCs than in normal HSCs (Fig. 2). The anti-apoptotic Bcl-2 family member proteinsm Bcl-2, Bcl-xL, Bcl-w, Mcl-1, Bfl1/A-1 and Bcl-B, bind to and inactivate $\mathrm{BH} 3$-domain pro-apoptotic proteins (45). High expression levels of these proteins have been found in various types of cancer and have been related to the development of chemoresistance in malignant tumor cells (45-47). In our study, we found that increased Bcl-xL expression, but not that of Bcl-2 or Mcl-1, caused an increase in resistance to doxorubicin in MPNST-derived SCs (Figs. 2 and 3). Manipulating Bcl-xL expression levels demonstrated that the reduced apoptosis sensitivity of MPNST-derived SCs was caused by Bcl-xL overexpression (Fig. 3).

Although NF1 LOH has been identified in benign neurofibromas (21-24), a much higher frequency of $N F 1 \mathrm{LOH}$ (>4-fold) has been observed in MPNSTs compared to neurofibromas in patients with NF1 (25). The interaction between $\mathrm{NF}^{-/-} \mathrm{SCs}$ and other types of $\mathrm{NF}^{+/-}$cells, including fibroblasts, mast cells and perineurial cells and the elevated expression of stem cell factors in $\mathrm{NF}^{-/-} \mathrm{SCs}$ in the tumor microenvironment has been implicated in the tumor progression of PNs to MPNSTs $(12,48)$. These results strongly indicate that the bi-allelic inactivation of the NF1 gene in SCs plays a crucial role in MPNST pathogenesis and NF1 tumorigenesis; however, little is known about the molecular mechanisms involved. Notably, NF1 deficiency promotes carcinogenesis by inducing heat shock factor 1 (HSF1), which is mediated by aberrant Ras/ MAPK signaling (49). HSF1 overexpression and activation has been observed in NF1-deficient MPNST cells and tumor resections from patients with NF1 (49). Intriguingly, as shown in a previous study, NF1 deficiency contributes to the epithelialmesenchymal transition (EMT) in NF1 (37). The expression levels of the EMT-related transcription factors, Snail, Twist and ZEB1, were significantly upregulated in the sNF96.2 MPNST-derived SCs compared with the normal HSCs. EMT is involved in cancer metastasis via the Ras/MAPK signaling pathway (50). Therefore, we investigated whether NF1 deficiency is directly involved in Bcl-xL upregulation in MPNST SCs. The results following manipulation of $N F 1$ expression levels demonstrated a close correlation between neurofibromin and Bcl-xL levels and sensitivity to doxorubicin-induced apoptosis in sNF96.2 SCs and HSCs (Figs. 4 and 5). Taken together, these results indicate that the high Bcl-xL expression in MPNST-derived SCs is caused by NF1 deficiency.

Neurofibromin depletion by NF1 siRNAs in HSCs resulted in Erk1/2 activation (Fig. 4A) and an increase in BCL2L1 mRNA levels (Fig. 4B). Treatment with the Erk1/2 inhibitor, PD98059, resulted in a slight increase in Bcl-xL levels in the neurofibromin-depleted HSCs (Fig. 6B). These results demonstrate that neurofibromin-mediated Bcl-xL expression is controlled at the transcriptional level via the Ras/MAPK signaling pathway. BCL2L1 gene transcription is regulated by a number of transcription factors, including Ets-1, Ets-2, Rel/ nuclear factor- $\kappa \mathrm{B}$, signal transducers and activators of transcription, activator protein 1 and $\mathrm{Spl}$ (51-53). Of note, these proteins are all downstream of the Ras-signaling pathway (54). NF1 deficiency-mediated Ras activation has been identified in a subpopulation of SCs $\left(N F 1^{-/-}\right)$but not fibroblasts $\left(N F 1^{-/}\right)$in mice with neurofibromas (55). Our results suggest that a neurofibromin deficiency in SCs caused by the bi-allelic inactivation at the NFI locus enhanced Ras signaling, which consequently led to the expression of $B C L 2 L 1$ transcription factors.

Bcl-xL overexpression contributes to the inhibtion of the effects of many chemotherapeutic drugs $(46,47)$. Reducing Bcl-xL expression restored apoptosis sensitivity to doxorubicin in sNF96.2 cells (Fig. 3), leading to a reasonable therapeutic strategy for patients with NF1 and MPNSTs through increased chemosensitization of malignant SCs by modulating the Bcl-xL expression level. ABT-737 selectively inhibits Bcl-2, Bcl-xL and Bcl-w (41). ABT-737 has been demonstrated to enhance synergistic chemosensitivity when used in combination with doxorubicin in other MPNST cells (33), chondrosarcoma cells (56), and hepatoblastoma cells (57). We thus examined apoptosis sensitivity of sNF96.2 MPNST-derived SCs by the combined treatment of ABT-737 and doxorubicin. As a result, ABT-737 synergistically enhanced sensitivity to doxorubicininduced apoptosis in sNF96.2 cells (Fig. 7). A very low dose of ABT-737 enhanced the cytotoxic effect of doxorubicin, and the concentrations required for approximately $50 \%$ cytotoxicity in sNF96.2 cells were $0.5 \mu \mathrm{g} / \mathrm{ml}$ doxorubicin and $0.1 \mu \mathrm{M}$ ABT-737. Notably, the concentrations of ABT-737 and doxo- 
rubicin required for effective apoptotic cell death were much lower in sNF96.2 cells than those in SNF02.2 cells, another MPNST SC line (33). sNF96.2 SCs are a NF1 LOH strain $\left(\mathrm{NFI}^{-/ /}\right)$, whereas sNF02.2 SCs harbor one intact $\mathrm{NF1}$ allele $\left(\mathrm{NFI}^{+/}\right)$, suggesting that combining ABT-737 and doxorubicin may increase the additive effects of the combined treatment in $N F 1^{-/-}$SCs. Considering that $N F 1^{-/-}$SCs play a major role in MPNST pathogenesis in NF1 $(31,48)$ and that a high frequency of $\mathrm{NF}^{-1-} \mathrm{SCs}$ is detected in NF1-associated MPNST tissues (25), ABT-737 and doxorubicin seems to be a good combination to effectively treat NF1-associated MPNSTs with minimal sideeffects. Although surgical resection is the primary treatment for MPNSTs, its limitation due to tumor location and tumor multiplicity has led to the development of a drug treatment approach. The proteins involved in the EGFR/Ras signaling and mTOR pathways have been the main chemotherapeutic targets for MPNSTs $(6,58)$. In our study, increased cell survival caused by the prevention of apoptosis was closely related to the chemoresistance in NF1-associated MPNSTs, suggesting that Bcl-xL may be good candidate for MPNST-targeted drug treatment.

In conclusion, we found the overexpression of the antiapoptotic protein, Bcl-xL, in MPNST tissues from patients with NF1 and in SCs derived from patients with NF1-associated MPNSTs. Our results demonstrate that the upregulation of Bcl-xL in MPNST-derived SCs was caused by NF1 deficiencymediated elevation in Ras/MAPK signaling. Our findings may provide an opportunity for the development of novel chemotherapeutic strategies for patients with NF1 and MPNSTs.

\section{Acknowledgements}

This study was supported by the Basic Science Research Program through the National Research Foundation of Korea (NRF) funded by the Ministry of Education, Science and Technology (2009-0093189).

\section{References}

1. Boyd KP, Korf BR and Theos A: Neurofibromatosis type 1. J Am Acad Dermatol 61: 1-14, 2009.

2. Ferner RE: Neurofibromatosis 1. Eur J Hum Genet 15: 131-138, 2007.

3. Cawthon RM, Weiss R, Xu GF, et al: A major segment of the neurofibromatosis type 1 gene: cDNA sequence, genomic structure, and point mutations. Cell 62: 193-201, 1990.

4. Jett K and Friedman JM: Clinical and genetic aspects of neurofibromatosis 1. Genet Med 12: 1-11, 2010.

5. Grobmyer SR, Reith JD, Shahlaee A, Bush CH and Hochwald SN: Malignant peripheral nerve sheath tumor: Molecular pathogenesis and current management considerations. J Surg Oncol 97: 340-349, 2008.

6. Gottfried ON, Viskochil DH, Fults DW and Couldwell WT: Molecular, genetic, and cellular pathogenesis of neurofibromas and surgical implications. Neurosurgery 58: 1-16, 2006.

7. Carroll SL and Stonecypher MS: Tumor suppressor mutations and growth factor signaling in the pathogenesis of NF1-associated peripheral nerve sheath tumors: II. The role of dysregulated growth factor signaling. J Neuropathol Exp Neurol 64: 1-9, 2005.

8. Kluwe L, Friedrich R and Mautner VF: Loss of NF1 allele in Schwann cells but not in fibroblasts derived from an NF1-associated neurofibroma. Genes Chromosomes Cancer 24: 283-285, 1999.

9. Rutkowski JL, Wu K, Gutmann DH, Boyer PJ and Legius E: Genetic and cellular defects contributing to benign tumor formation in neurofibromatosis type 1. Hum Mol Genet 9: 1059-1066, 2000.
10. Serra E, Rosenbaum T, Winner U, et al: Schwann cells harbor the somatic NF1 mutation in neurofibromas: evidence of two different Schwann cell subpopulations. Hum Mol Genet 9: 3055-3064, 2000.

11. Zhu Y, Ghosh P, Charnay P, Burns DK and Parada LF: Neurofibromas in NF1: Schwann cell origin and role of tumor environment. Science 296: 920-922, 2002.

12. Jouhilahti EM, Peltonen S, Heape AM and Peltonen J: The pathoetiology of neurofibromatosis 1. Am J Pathol 178: 1932-1939, 2011.

13. Staser K, Yang FC and Clapp DW: Mast cells and the neurofibroma microenvironment. Blood 116: 157-164, 2010.

14. Katz D, Lazar A and Lev D: Malignant peripheral nerve sheath tumour (MPNST): the clinical implications of cellular signalling pathways. Expert Rev Mol Med 11: e30, 2009.

15. Brems H, Beert E, de Ravel T and Legius E: Mechanisms in the pathogenesis of malignant tumours in neurofibromatosis type 1 . Lancet Oncol 10: 508-515, 2009.

16. Spurlock G, Knight SJ, Thomas N, Kiehl TR, Guha A and Upadhyaya M: Molecular evolution of a neurofibroma to malignant peripheral nerve sheath tumor (MPNST) in an NF1 patient: correlation between histopathological, clinical and molecular findings. J Cancer Res Clin Oncol 136: 1869-1880, 2010.

17. Evans DG, Baser ME, McGaughran J, Sharif S, Howard E and Moran A: Malignant peripheral nerve sheath tumours in neurofibromatosis 1. J Med Genet 39: 311-314, 2002.

18. McCaughan JA, Holloway SM, Davidson R and Lam WW: Further evidence of the increased risk for malignant peripheral nerve sheath tumour from a Scottish cohort of patients with neurofibromatosis type 1. J Med Genet 44: 463-466, 2007.

19. Tucker T, Wolkenstein P, Revuz J, Zeller J and Friedman JM: Association between benign and malignant peripheral nerve sheath tumors in NF1. Neurology 65: 205-211,2005.

20. McQueen M, MacCollin M, Gusella J and Plotkin SR: Patient and physician attitudes regarding clinical trials in neurofibromatosis 1. J Neurosci Nurs 40: 341-345, 2008.

21. Sawada S, Florell S, Purandare SM, Ota M, Stephens K and Viskochil D: Identification of NF1 mutations in both alleles of a dermal neurofibroma. Nat Genet 14: 110-112, 1996.

22. Wiest V, Eisenbarth I, Schmegner C, Krone W and Assum G: Somatic NF1 mutation spectra in a family with neurofibromatosis type 1: toward a theory of genetic modifiers. Hum Mutat 22: 423-427, 2003.

23. Kluwe L, Friedrich RE and Mautner VF: Allelic loss of the NF1 gene in NF1-associated plexiform neurofibromas. Cancer Genet Cytogenet 113: 65-69, 1999.

24. Rasmussen SA, Overman J, Thomson SA, et al: Chromosome 17 loss-of-heterozygosity studies in benign and malignant tumors in neurofibromatosis type 1 . Genes Chromosomes Cancer 28: $425-431,2000$

25. Upadhyaya M, Kluwe L, Spurlock G, et al: Germline and somatic NF1 gene mutation spectrum in NF1-associated malignant peripheral nerve sheath tumors (MPNSTs). Hum Mutat 29: 74-82, 2008

26. Watanabe T, Oda Y, Tamiya S, Masuda K and Tsuneyoshi M: Malignant peripheral nerve sheath tumour arising within neurofibroma. An immunohistochemical analysis in the comparison between benign and malignant components. J Clin Pathol 54: 631-636, 2001.

27. Miller SJ, Rangwala F, Williams J, et al: Large-scale molecular comparison of human schwann cells to malignant peripheral nerve sheath tumor cell lines and tissues. Cancer Res 66: 2584-2591, 2006

28. Mantripragada KK, Spurlock G, Kluwe L, et al: High-resolution DNA copy number profiling of malignant peripheral nerve sheath tumors using targeted microarray-based comparative genomic hybridization. Clin Cancer Res 14: 1015-1024, 2008.

29. Upadhyaya M, Spurlock G, Thomas L, et al: Microarray-based copy number analysis of neurofibromatosis type-1 (NF1)associated malignant peripheral nerve sheath tumors reveals a role for Rho-GTPase pathway genes in NF1 tumorigenesis. Hum Mutat 33: 763-776, 2012.

30. Upadhyaya M: Genetic basis of tumorigenesis in NF1 malignant peripheral nerve sheath tumors. Front Biosci 16: 937-951, 2011.

31. Carroll SL: Molecular mechanisms promoting the pathogenesis of Schwann cell neoplasms. Acta Neuropathol 123: 321-348, 2012.

32. Woodruff JM: Pathology of tumors of the peripheral nerve sheath in type 1 neurofibromatosis. Am J Med Genet 89: 23-30, 1999. 
33. Lee SJ, Park HJ, Kim YH, et al: Inhibition of Bcl-xL by ABT-737 enhances chemotherapy sensitivity in neurofibromatosis type 1-associated malignant peripheral nerve sheath tumor cells. Int J Mol Med 30: 443-450, 2012

34. Jeong SY, Gaume B, Lee YJ, et al: Bcl-x(L) sequesters its C-terminal membrane anchor in soluble, cytosolic homodimers. EMBO J 23: 2146-2155, 2004.

35. Ferner RE, Huson SM, Thomas N, et al: Guidelines for the diagnosis and management of individuals with neurofibromatosis 1. J Med Genet 44: 81-88, 2007.

36. Weiss SW, Langloss JM and Enzinger FM: Value of S-100 protein in the diagnosis of soft tissue tumors with particular reference to benign and malignant Schwann cell tumors. Lab Invest 49: 299-308, 1983

37. Arima Y, Hayashi H, Kamata K, et al: Decreased expression of neurofibromin contributes to epithelial-mesenchymal transition in neurofibromatosis type 1. Exp Dermatol 19: e136-e141, 2010.

38. McCubrey JA, Steelman LS, Chappell WH, et al: Roles of the $\mathrm{Raf} / \mathrm{MEK} / \mathrm{ERK}$ pathway in cell growth, malignant transformation and drug resistance. Biochim Biophys Acta 1773: 1263-1284, 2007.

39. Moretti VM, Crawford EA, Staddon AP, Lackman RD and Ogilvie CM: Early outcomes for malignant peripheral nerve sheath tumor treated with chemotherapy. Am J Clin Oncol 34: 417-421, 2011.

40. Hiatt KK, Ingram DA, Zhang Y, Bollag G and Clapp DW: Neurofibromin GTPase-activating protein-related domains restore normal growth in $\mathrm{Nf1}^{-/}$cells. J Biol Chem 276: 7240-7245, 2001.

41. Ni Chonghaile T and Letai A: Mimicking the BH3 domain to kill cancer cells. Oncogene 27 (Suppl 1): S149-S157, 2008.

42. Brossier NM and Carroll SL: Genetically engineered mouse models shed new light on the pathogenesis of neurofibromatosis type I-related neoplasms of the peripheral nervous system. Brain Res Bull 88: 58-71, 2012.

43. Chai G, Liu N, Ma J, et al: MicroRNA-10b regulates tumorigenesis in neurofibromatosis type 1. Cancer Sci 101: 1997-2004, 2010.

44. Subramanian S, Thayanithy V, West RB, et al: Genome-wide transcriptome analyses reveal p53 inactivation mediated loss of miR-34a expression in malignant peripheral nerve sheath tumours. J Pathol 220: 58-70, 2010.

45. Kang $\mathrm{MH}$ and Reynolds $\mathrm{CP}$ : Bcl-2 inhibitors: targeting mitochondrial apoptotic pathways in cancer therapy. Clin Cancer Res 15: 1126-1132, 2009.
46. Kostanova-Poliakova D and Sabova L: Anti-apoptotic proteinstargets for chemosensitization of tumor cells and cancer treatment. Neoplasma 52: 441-449, 2005.

47. Karnak D and Xu L: Chemosensitization of prostate cancer by modulating Bcl-2 family proteins. Curr Drug Targets 11: 699-707, 2010

48. Le LQ and Parada LF: Tumor microenvironment and neurofibromatosis type I: connecting the GAPs. Oncogene 26: 4609-4616, 2007.

49. Dai C, Santagata S, Tang Z, et al: Loss of tumor suppressor NF1 activates HSF1 to promote carcinogenesis. J Clin Invest 122: 3742-3754, 2012.

50. Edme N, Downward J, Thiery JP and Boyer B: Ras induces NBT-II epithelial cell scattering through the coordinate activities of Rac and MAPK pathways. J Cell Sci 115: 2591-2601, 2002

51. Sevilla L, Zaldumbide A, Pognonec P and Boulukos KE: Transcriptional regulation of the bcl-x gene encoding the antiapoptotic Bcl-xL protein by Ets, Rel/NFkappaB, STAT and AP1 transcription factor families. Histol Histopathol 16: 595-601, 2001.

52. Lee J, Kannagi M, Ferrante RJ, Kowall NW and Ryu H: Activation of Ets- 2 by oxidative stress induces Bcl-xL expression and accounts for glial survival in amyotrophic lateral sclerosis. FASEB J 23: 1739-1749, 2009.

53. Grad JM, Zeng XR and Boise LH: Regulation of Bcl-xL: a little bit of this and a little bit of STAT. Curr Opin Oncol 12: 543-549, 2000.

54. McCubrey JA, Steelman LS, Abrams SL, et al: Roles of the RAF/ MEK/ERK and PI3K/PTEN/AKT pathways in malignant transformation and drug resistance. Adv Enzyme Regul 46: 249-279, 2006.

55. Sherman LS, Atit R, Rosenbaum T, Cox AD and Ratner N: Single cell Ras-GTP analysis reveals altered Ras activity in a subpopulation of neurofibroma Schwann cells but not fibroblasts. J Biol Chem 275: 30740-30745, 2000

56. van Oosterwijk JG, Herpers B, Meijer D, et al: Restoration of chemosensitivity for doxorubicin and cisplatin in chondrosarcoma in vitro: BCL-2 family members cause chemoresistance. Ann Oncol 23: 1617-1626, 2012.

57. Lieber J, Kirchner B, Eicher C, et al: Inhibition of Bcl-2 and Bcl-X enhances chemotherapy sensitivity in hepatoblastoma cells. Pediatr Blood Cancer 55: 1089-1095, 2010.

58. Gottfried ON, Viskochil DH and Couldwell WT: Neurofibromatosis type 1 and tumorigenesis: molecular mechanisms and therapeutic implications. Neurosurg Focus 28: E8, 2010. 